\title{
MODELING OF COALESCENCE IN TURBULENT GAS-DROPLET FLOWS
}

\author{
P. VILLEDIEU* AND O. SIMONIN ${ }^{\dagger}$
}

\begin{abstract}
The present paper is devoted to the kinetic modeling of coalescence in turbulent gas-droplet flows. A new approach is proposed for the calculation of the collision probability, that takes into account the correlations induced by the effect of the gas on the droplet motion. The key ingredient is to replace the simple distribution function $f_{p}^{(1)}(t, \mathbf{x}, \mathbf{v}, r)$, which is classically used for the description of a spray at the kinetic level, by the joint distribution function, $f_{p g}^{(1)}(t, \mathbf{x}, \mathbf{v}, \mathbf{u}, r)$, which explicitely depends on the fluctuating gas velocity $\mathbf{u}$ at the droplet position.
\end{abstract}

\section{NOtATIONS}

$\tau_{p} \quad$ Response time of a droplet to the drag force

$\tau_{g} \quad$ Autocorrelation time scale of the turbulence along droplet trajectories

$T_{L} \quad$ Lagrangian integral time scale of the turbulence

$\rho_{g} \quad$ Gas density

$\rho_{l} \quad$ Liquid density

$\mu_{g} \quad$ Dynamic viscosity of the gas

$k_{g} \quad$ Turbulent kinetic energy of the gas

$\sigma_{g} \quad$ Turbulence intensity

$\varepsilon_{g} \quad$ Turbulence dissipation rate

$r \quad$ Droplet radius

v Droplet velocity

$\mathbf{u} \quad$ Fluctuating gas velocity seen by a droplet

$\mathbf{u}_{g} \quad$ Turbulent velocity field of the gas

$\mathbf{U}_{g} \quad$ Mean velocity field of the gas

$\mathbb{E} \quad$ Phase space

$S \quad$ Stokes number

$f_{p g}^{(1)}, f_{p g}$ One-particle joint gas-droplet distribution function

$f_{p g}^{(2)} \quad$ Two-particle joint gas-droplet distribution function

$f_{p}^{(1)}, f_{p} \quad$ One-particle droplet distribution function

$p_{g} \quad$ Eulerian gas pdf

$b \quad$ Collision impact parameter

We Collision Weber number

$R e, R \quad$ Reynolds number

$\Delta \quad$ Colliding droplet size ratio

\section{Introduction}

This paper deals with the statistical modeling of two-phase flows consisting of droplets immersed in a gas. The numerical simulation of such flow is now currently

\footnotetext{
*ONERA, 2 Avenue Ed. Belin, 31055 Toulouse, France (philippe.villedieu@onera.fr).

†IMFT, UMR CNRS, Allée du Professeur C. Soula, 31000 Toulouse, France (simonin@imft.fr).
} 


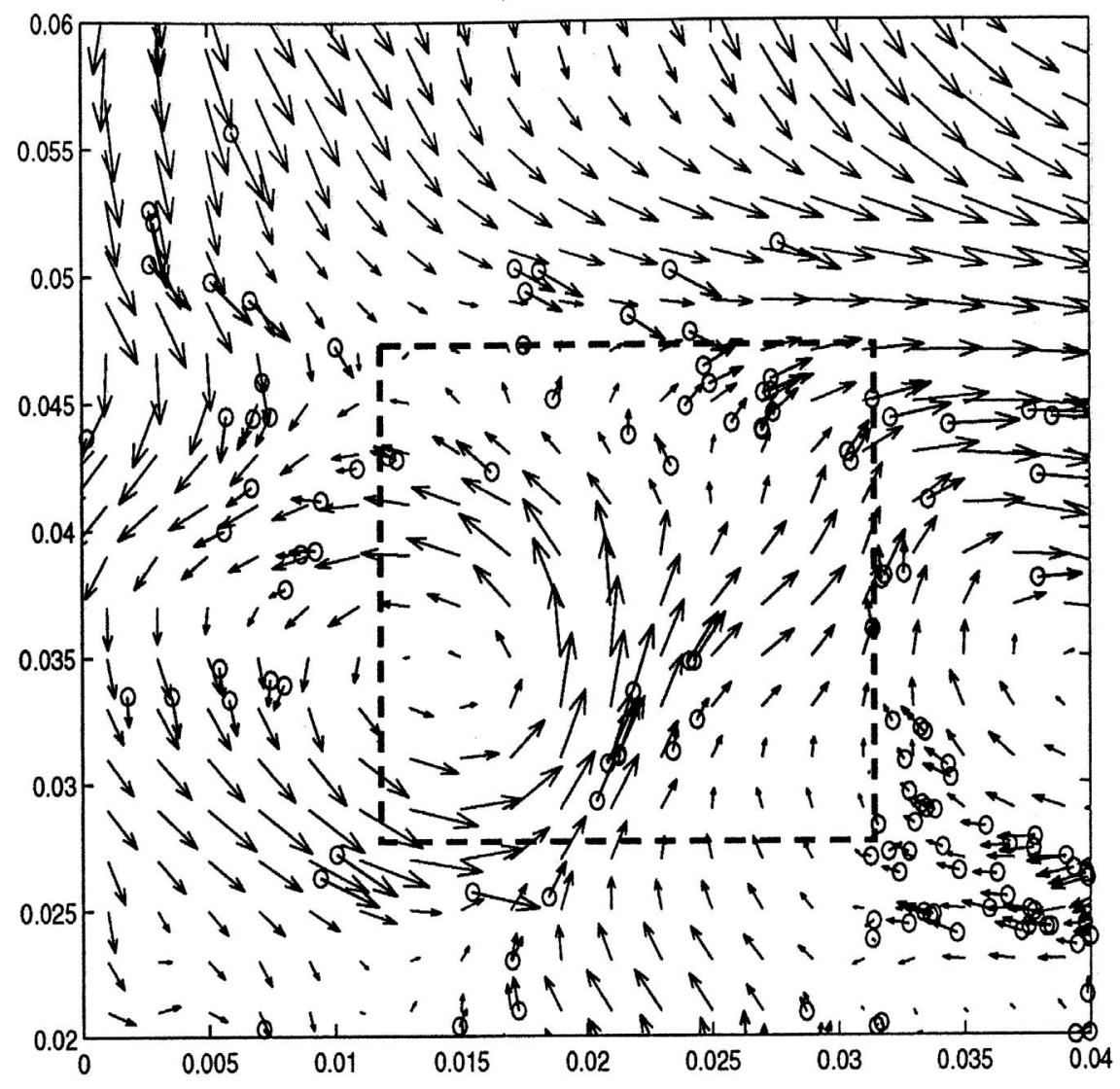

(a)

Case of small droplets

FIG. 1.1. Instantaneous droplet velocity field issued from a DNS calculation. (P. Fevrier thesis dissertation, 2000)

used in a lot of applications such as spray combustion in Diesel or aeronautic engines $[25,22]$, performance optimization of solid rocket motors with aluminum particles inside the propellant [15], prediction of rain drop formation, .... The accurate modeling of droplet collision and coalescence is very important because collision may have a significant impact on the mean droplet size [26] and also on the dispersion and velocities of the drops [12]. For example, in a combustion chamber, the lifetime of a droplet depends on the square of its diameter. Therefore size increasing may have a great influence of the mixing between air and vapor and in turn on the global com- 


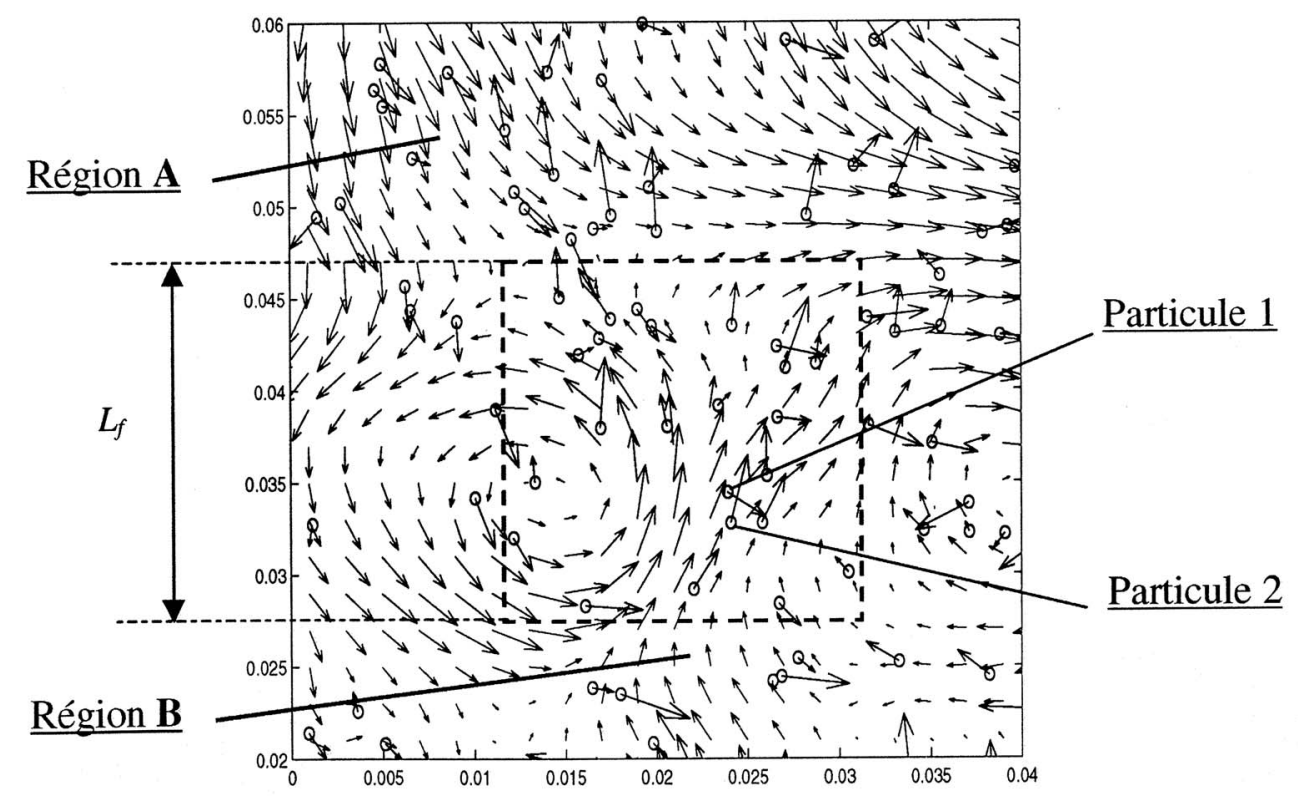

Case of large droplets

FIG. 1.2. Instantaneous droplet velocity field issued from a DNS calculation. (P. Fevrier thesis dissertation, 2000)

bustion rate.

Typically, a drop collision model is divided in two parts: first, a rule to compute the collision probability and second, a rule to calculate the outcome of the collision. In this paper we present a review of some existing models for the second point and we propose a new approach for the first one, in the generic case of homogeneous turbulent flows. All the commonly used droplet interaction models [26, 30, 15, 29] are based upon the hypothesis that before a collision, the droplet velocities are uncorrelated (chaos assumption). Hence the collision probability is calculated exactly in the same way as for molecules in rarefied gas dynamics. However, in a turbulent gas flow, this assumption is generally not verified at all, especially for small droplets, whose velocity is strongly correlated with the gas motion, as it can be observed on figures 1.1 and 1.2 (DNS results from P. Février thesis dissertation, 2000 [11]). This is due to the fact that small droplets have a very low inertia and follow almost the same trajectories as gas particles.

The main objective of the present paper is to propose a new approach for the calculation of the collision probability, which takes into account the correlations induced by the action of the gas on the droplet motion. The key ingredient is to replace the one-particle distribution function $f_{p}^{(1)}(t, \mathbf{x}, \mathbf{v}, r)$, which is classically used for the description of the spray at the kinetic level, by the one-particle joint distribution function, $f_{p g}^{(1)}(t, \mathbf{x}, \mathbf{v}, \mathbf{u}, r)$, which explicitely depends on the fluctuating gas velocity $\mathbf{u}$ at the droplet position. This idea was first introduced by Simonin for eulerian modeling of turbulent granular flows [27]. This new approach can be considered 
as a generalization of the usual framework, in the sense that the standard collision model can be recovered if gas and droplet velocities are supposed to be uncorrelated: $f_{p g}^{(1)}(t, \mathbf{x}, \mathbf{v}, \mathbf{u}, r)=f_{p}^{(1)}(t, \mathbf{x}, \mathbf{v}, r) p_{g}(t, \mathbf{x}, \mathbf{u})$.

The paper is organized as follows. In section 2 , we introduce the general form of the kinetic equation, based on the joint distribution function, for turbulent gasdroplet flows. The third section is devoted to the derivation of the collision model. In the last part, we focus on dilute sprays, for which the mean collision time is large with respect to the typical time scale of the turbulence. We show that it is possible to derive a simplified asymptotic model of Smoluchovski type [28], that could be used to provide reference numerical solutions, which in turn could be compared with results issued from direct or Monte-Carlo numerical simulations.

\section{Statistical modeling of turbulent sprays}

2.1. Droplet motion equations. For the sake of simplicity, we shall assume the following hypotheses on the gas velocity field:

- (i) the average gas velocity $\mathbf{U}_{g}$ is zero;

- (ii) the statistical properties of the turbulent velocity field, $\mathbf{u}_{g}(t, \mathbf{x})$, are stationary, isotropic and spatially homogeneous; in particular the turbulent kinetic energy $k_{g}$ and the mean dissipation rate $\varepsilon_{g}$ are two given constants;

- (iii) there is no influence of the droplets on the gas motion (one-way coupling);

- (iv) for all droplets, their diameter is lower than the Kolmogorov turbulence length scale $\eta_{K}$, hence the gas velocity at the droplet position is defined without ambiguity.

For large liquid to gas density ratio $\left(\rho_{l} \gg \rho_{g}\right)$, the drag force is dominant and the equation of motion for a droplet writes

$$
\left\{\begin{array}{l}
\frac{d \mathbf{x}}{d t}(t)=\mathbf{v}(t) \\
\frac{d \mathbf{v}}{d t}(t)=\frac{\mathbf{u}_{g}(t, \mathbf{x}(t))-\mathbf{v}(t)}{\tau_{p}}
\end{array}\right.
$$

where $\mathbf{u}_{g}(t, \mathbf{x}(t))$ is the instantaneous gas velocity at the droplet position and $\tau_{p}$ stands for the particle response time. In the limit of small Reynolds number $(R e=$ $\left.\frac{2 \rho_{g}|\mathbf{v}-\mathbf{u}| r}{\mu_{g}} \ll 1\right), \tau_{p}$ only depends on the particle radius and is given by

$$
\tau_{p}=\frac{2 \rho_{l} r^{2}}{9 \mu_{g}} .
$$

The major difficulty comes from the turbulent velocity field $\mathbf{u}_{g}(t, \mathbf{x})$ being only known through its statiscal properties. Hence, a model must be used to "sample" the gas velocity, $\mathbf{u}_{g}(t, \mathbf{x}(t))$ seen by a droplet along its trajectory. In the case of homogeneous isotropic flows, by analogy with the work of Pope et al [21] on lagrangian turbulence modeling, Pozorsky, Minier and Simonin have suggested to use the following stochastic differential equation $[23,18,19]$ :

$$
d \mathbf{u}=-\frac{\mathbf{u}}{\tau_{g}} d t+\sqrt{\frac{2 \sigma_{g}^{2}}{\tau_{g}}} d \mathbf{W}_{\mathbf{t}}
$$




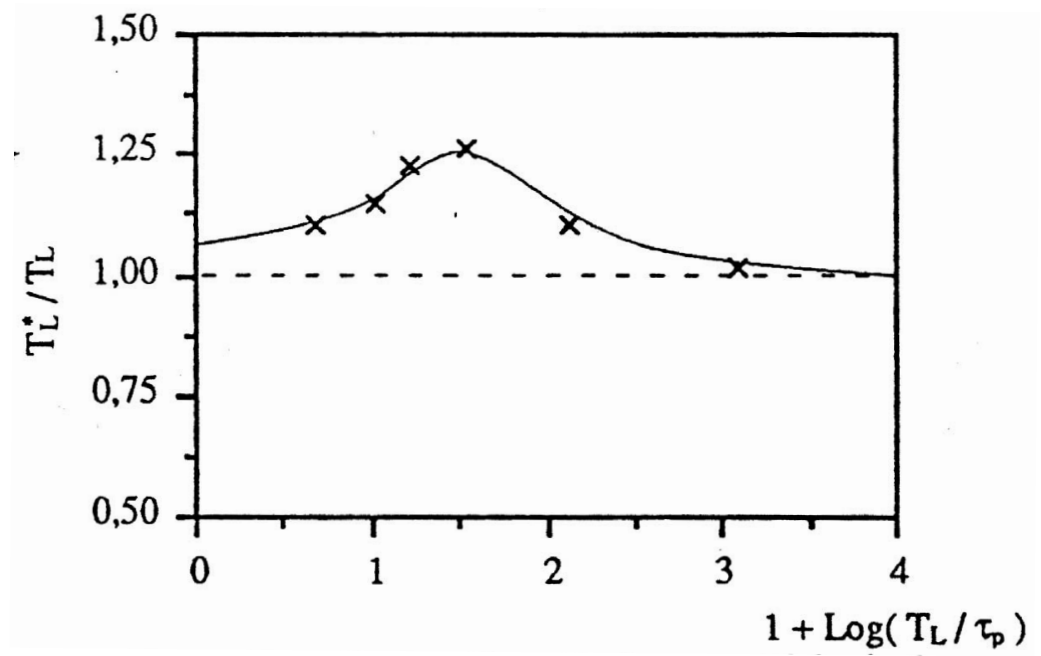

FIG. 2.1. Turbulent time scale ratio (Deutsch et al, 1992)

where $\mathbf{W}_{\mathbf{t}}=\left(\mathbf{W}_{\mathbf{t}, 1}, \mathbf{W}_{\mathbf{t}, 2}, \mathbf{W}_{\mathbf{t}, 3}\right)$ is the Wiener process on $\mathbb{R}^{3}$ with $<d \mathbf{W}_{\mathbf{t}, i} d \mathbf{W}_{\mathbf{t}, j}>=$ $\delta_{i j} d t, \sigma_{g}=\frac{2 k_{g}}{3}$ is the turbulence intensity and $\tau_{g}$ is a positive constant whose physical meaning is precised hereafter. For simplicity, assume that the initial condition of (2.3) is a Gaussian random vector; then the solution is a stationary Gaussian Markov process (known as the Ornstein-Uhlenbeck process) and is completely characterized by its mean $\langle\mathbf{u}(t)\rangle=0$, its variance $\left\langle\mathbf{u}^{2}(t)\right\rangle=3 \sigma_{g}^{2}$ and its autocorrelation function, which writes

$$
r(s)=\frac{<\mathbf{u}(t+s) \cdot \mathbf{u}(t)>}{3 \sigma_{g}^{2}}=\exp \left(-s / \tau_{g}\right) .
$$

This last equation shows that the parameter $\tau_{g}=\int_{0}^{+\infty} r(s) d s$ represents the time scale during which the values of the gas velocity "seen" by a droplet along its path remain strongly correlated. We refer to the surveys of Pope [21] or Minier and Peirano [19] for a deeper insight into the physical relevance of equation (2.3).

In this work, for the sake of simplicity, we shall assume that $\tau_{g}$ is a constant only depending on $k_{g}$ and $\varepsilon_{g}$, according to the relation:

$$
\tau_{g}=\frac{4}{3 C_{0}} \frac{k_{g}}{\varepsilon_{g}}
$$

where $C_{0}$ is a given constant, lying in the range 5.0 - 6.5 [21]. Equation (2.5) corresponds to the assumption that the integral time scale of the turbulence along droplet and gas particle trajectories are the same. Rigorously, this hypothesis is only justified in the case of very small droplets for which the crossing trajectory effect is negligible. For heavy particles, using LES computations (see figure 3), Deutsch et al [9] have shown that, in absence of gravity, the ratio of $T_{L}^{*}=\tau_{g}$ to the Lagrangian time scale of 
the gas turbulence $T_{L}$ remains in the range 1.0 - 1.25 and reveals a bell-shaped curve. One can observe on figure 2.1 that the two asymptotic limits are correctly reproduced by the numerical results: very small particles follow the gas, hence $\tau_{g} \simeq T_{L}$, while heavy particles tend to remain at rest and to see the eulerian time scale of the turbulence $T_{E}$ which is about 1.08 times greater than the lagrangian one. Improved model, based on a dependance of $\tau_{g}$ on the relative velocity $|\mathbf{v}-\mathbf{u}|$, have been proposed in the literature. For details on this subject, we refer the reader to the works of Csanady [5], Pozorsky et al [23, 18] or to the review paper of Minier and Peirano [19].

Finally, in this paper, we shall assume that droplet motion is governed by the following set of differential equations:

$$
\left\{\begin{array}{l}
\frac{d \mathbf{x}}{d t}=\mathbf{v} \\
\frac{d \mathbf{v}}{d t}=\frac{\mathbf{u}-\mathbf{v}}{\tau_{p}(r)} \\
d \mathbf{u}=-\frac{\mathbf{u}}{\tau_{g}} d t+\sqrt{\frac{2 \sigma_{g}^{2}}{\tau_{g}}} d W_{t}
\end{array}\right.
$$

where $\tau_{p}$ and $\tau_{g}$ are respectively defined by (2.2) and (2.5).

2.2. Joint density equation. Let us now introduce the one-particle joint density function $f_{p g}^{(1)}$ (also denoted by $f_{p g}$ for simplicity's sake) which is defined as follows: for any subset $\Omega=\Pi_{x} \times \Pi_{v} \times \Pi_{u} \times I_{r}$ of the phase space $\mathbb{E}=\mathbb{R}_{x}^{3} \times \mathbb{R}_{v}^{3} \times \mathbb{R}_{u}^{3} \times \mathbb{R}_{r}^{+}$, the integral

$$
\int_{\Pi_{x}} \int_{\Pi_{v}} \int_{\Pi_{u}} \int_{I_{r}} f_{p g}(t, \mathbf{x}, \mathbf{v}, \mathbf{u}, r) d \mathbf{x} d \mathbf{v} d \mathbf{u} d r
$$

represents the mean number of droplets (in a statistical sense) whose radius lies in the interval $I_{r}$, located in $\Pi_{x}$, having their velocity $\mathbf{v}$ in the set $\Pi_{v}$ and "seeing" a value of the fluctuating gas velocity which belongs to the range $\Pi_{u}$.

If, as a first step, droplet collisions are not taken into account, it follows from system (2.6) and from Kolmogorov theorem that $f_{p g}$ satisfies the following FokkerPlanck equation:

$$
\partial_{t} f_{p g}+\operatorname{div}_{x}\left(\mathbf{v} f_{p g}\right)+\operatorname{div}_{v}\left(\frac{\mathbf{u}-\mathbf{v}}{\tau_{p}} f_{p g}\right)-\operatorname{div}_{u}\left(\frac{\mathbf{u}}{\tau_{g}} f_{p g}+\frac{\sigma_{g}^{2}}{\tau_{g}} \nabla_{u} f_{p g}\right)=0
$$

We note that the effect of the turbulence on the droplet distribution function is taken into account through the presence of the second order diffusion operator $D\left(f_{p g}\right)=-\operatorname{div}_{u}\left(\frac{\mathbf{u}}{\tau_{g}} f_{p g}+\frac{\sigma_{g}^{2}}{\tau_{g}} \nabla_{u} f_{p g}\right)$ in the l.h.s. of the kinetic equation. In the usual framework, based on the simple distribution function $f_{p}(t, \mathbf{x}, \mathbf{v}, r)$, the corresponding equation writes: 


$$
\partial_{t} f_{p}+\operatorname{div}_{x}\left(\mathbf{v} f_{p}\right)+\operatorname{div}_{v}\left(\frac{-\mathbf{v}}{\tau_{p}} f_{p}\right)+D\left(f_{p}\right)=0
$$

with $D$ being a second order diffusion operator with respect to the variable $\mathbf{v}$, whose expression depends on the hypothesis that has been used to model the turbulent motion of the droplets in the gas. About this problem and related questions, we refer to the papers of Reeks [24], Clouet and Domelevo [6] or Poupaud and Goudon [13]. Note also that, by using asymptotic analysis, a reduced kinetic equation, of the same form as (2.8), can be formally derived from (2.7) [7].

When collision effects are accounted for, equation (2.7) becomes:

$$
\begin{gathered}
\partial_{t} f_{p g}+\operatorname{div}_{x}\left(\mathbf{v} f_{p g}\right)+\operatorname{div}_{v}\left(\frac{\mathbf{u}-\mathbf{v}}{\tau_{p}} f_{p g}\right) \\
-\operatorname{div}_{u}\left(\frac{\mathbf{u}}{\tau_{g}} f_{p g}+\frac{\sigma_{g}^{2}}{\tau_{g}} \nabla_{u} f_{p g}\right)=Q_{\text {coll }}\left(f_{p g}^{(2)}\right)
\end{gathered}
$$

where $Q_{\text {coll }}$ is the collision operator, for which an expression will be given in the next section, and $f_{p g}^{(2)}$ is the two-particle joint density function defined as follows: the integral

$$
\int_{\Omega} \int_{\Omega_{*}} f_{p g}^{(2)}\left(t, \xi, \xi_{*}\right) d \xi_{*} d \xi
$$

represents the averaged number of droplet pairs such that the first droplet lies in $\Omega$ and the second one is in $\Omega_{*}$.

At this stage, equation (2.9) is not closed. A modeling assumption is needed to give a relation between $f_{p g}^{(2)}$ and $f_{p g}^{(1)}$. This problem will be adressed in the next section.

\section{Collision modeling}

In this section, we first present a review of some classical models, coming from the literature, in order to predict the outcome of a collision and to calculate the collisional cross section. Then the problem of finding a relation between $f_{p g}^{(2)}$ and $f_{p g}^{(1)}$ is addressed and we show, that in the more general framework based on the joint distribution function, it is possible to derive an approximated closure relation, which does not implicitely assume that gas and droplet velocities are uncorrelated.

3.1. Collision outcome. Experimental observations with water droplets $[4,1,10]$ have shown that a binary collision may lead either to permanent coalescence or to temporary coalescence followed by separation and sometimes by the formation of satellite droplets. Up to now, the existing models to predict the outcome of a collision are all based on experimental data combined with a simplified theoretical approach. They make use of three dimensionless parameters:

- the Weber number based on the relative velocity between the colliding droplets:

$W e=\frac{\rho_{l}\left|\mathbf{v}-\mathbf{v}_{*}\right| r^{*}}{\sigma_{l}}$,

- the impact parameter $b=\frac{I}{r+r_{*}}$, 
- the droplet size ratio $\Delta=\frac{r_{*}}{r}$,

where we have adopted the convention $r_{*}=\min \left(r, r_{*}\right)$. The result of a collision is mainly conditioned by the balance between inertial and capillary forces. For a grazing or a high speed head on collision, surface tension is not strong enough to permanently maintain the two droplets together, hence the collision is followed by a separation. Conversely, for moderate values of the relative velocity and impact parameter, the collision will lead to permanent coalescence. According to the experiments of BrazierSmith et al [4] or Ashgriz and Poo [1], the limits between these different regimes can be characterized by two empirical functions, denoted by $b_{1}(W e, \Delta)$ and $b_{2}(W e, \Delta)$, and defined as follows:

- if $b<b_{1}(W e, \Delta)$ or $b>b_{2}(W e, \Delta)$ the collision results in a temporary coalescence followed by the separation of the two droplets; the first case is called reflexive separation and the second one stretching separation;

- if $b_{1}(W e, \Delta)<b<b_{2}(W e, \Delta)$, the collision results in a permanent coalescence.

It is worth mentioning that for low values of $\Delta$ (collision between droplets of very different sizes) collision almost always results in a permanent coalescence; it means that $b_{1} \rightarrow 0$ and $b_{2} \rightarrow 1$ when $\Delta$ goes to 0 . For the sake of completeness, we recall here the expressions of $b_{1}$ and $b_{2}$ proposed by Brazier-Smith et al in [4]. They correspond to the simplest and most frequently used model.

$$
\left\{\begin{array}{l}
b_{1}^{B S}(W e, \Delta)=0 \\
b_{2}^{B S}(W e, \Delta)=\operatorname{Min}\left(1, \sqrt{\frac{24}{5 W e}} \frac{\left(1+\Delta^{3}\right)^{11 / 6}}{(1+\Delta) \Delta^{5 / 2}}\left[1+\Delta^{2}-\left(1+\Delta^{3}\right)^{2 / 3}\right]^{1 / 2}\right)
\end{array}\right.
$$

In the experiments performed by Brazier-Smith et al, high speed collisions were not studied, This is why they did not observe the case of reflexive separation and found $b_{1}=0$. We refer to [1] and [10] for other expressions of $b_{1}$ and $b_{2}$ with a largest range of application.

The second problem to solve in order to characterize the outcome of a binary collision is the determination of the post-collisional velocity and radius of the resulting droplets. According to the experimental results of Estrade et al [10], it appears that a grazing collision followed by a stretching separation does not significantly modify the initial droplet velocities. Therefore, if the satellite droplets which may be produced by the collision are ignored, it can be assumed, with an acceptable accuracy, that:

$$
\left\{\begin{array}{ll}
\mathbf{v}^{\prime \prime}=\mathbf{v}, & r "=r, \\
\mathbf{v}_{*}=\mathbf{v}_{*}, & r_{*}=r_{*},
\end{array} \quad\right. \text { stretching separation regime. }
$$

The case of head on collisions leading to a reflexive separation is more complex because the post-collisional velocities generally differ a lot from the initial ones. If the production of satellite droplets is ignored again and if the total momentum of the colliding droplets is supposed to be conserved, the following model may be used: 


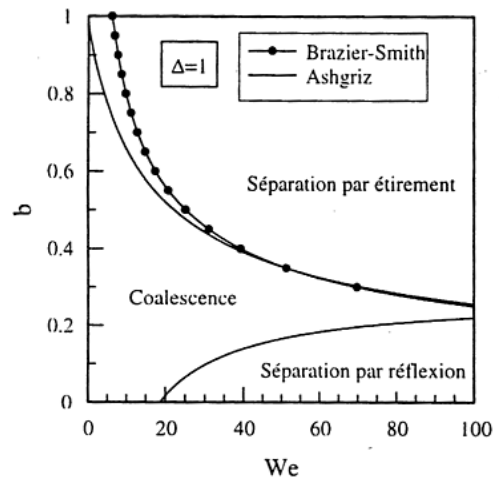

a) Case: $\Delta=1$

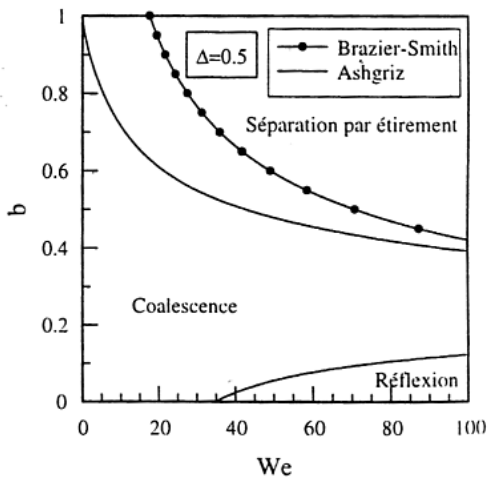

b) Case: $\Delta=0.5$

FIG. 3.1. Comparison of Brazier-Smith et al (solid line) and Ashgriz-Poo (dashed line) models for the calculation of functions $b_{1}$ and $b_{2}$

$$
\left\{\begin{array}{l}
\mathbf{v}^{\prime \prime}=\mathbf{v}-\frac{2 e r_{*}^{3}}{r^{3}+r_{*}^{3}}\left(\mathbf{v}-\mathbf{v}_{\star}\right) \cdot \mathbf{n} \mathbf{n}, \quad r "=r, \\
\mathbf{v}_{\star} "=\mathbf{v}_{\star}+\frac{2 e r^{3}}{r^{3}+r_{*}^{3}}\left(\mathbf{v}-\mathbf{v}_{\star}\right) \cdot \mathbf{n} \mathbf{n}, \quad r_{*}^{\prime \prime}=r_{*},
\end{array} \quad\right. \text { reflexive separation regime, }
$$

where $\mathbf{n}$ denotes the unit vector along the direction of the droplet center line and $e \in] 1 / 2,1]$ denotes the inelasticity parameter of the collision; it is related to the amount of energy dissipated during the temporary coalescence and can be estimated from experimental data.

Lastly, in case of permanent coalescence, the post-collisional values of the velocity and radius of the outcoming droplet are deduced from the conservation of total mass and momentum:

$$
\left\{\begin{array}{l}
\mathbf{v}_{\#}=\frac{r^{3} \mathbf{v}}{r^{3}+r_{*}^{3}}+\frac{r_{*}^{3} \mathbf{v}_{*}}{r^{3}+r_{*}^{3}} \quad \text { coalescence regime. } \\
r_{\#}=\left(r^{3}+r_{*}^{3}\right)^{1 / 3}
\end{array}\right.
$$

3.2. Collisional cross section. For hard spheres in vacuum, the collisional cross section writes: $\sigma=\pi\left(r+r_{*}\right)^{2}$. In the case of droplets, this expression must be modified to take into account the effect of the surrounding gas flow. It is replaced by:

$$
\sigma=\pi E_{\text {coll }}\left(r, r_{*},\left|\mathbf{v}-\mathbf{v}_{*}\right|,|\mathbf{v}-\mathbf{u}|\right)\left(r+r_{*}\right)^{2}
$$

where $E_{\text {coll }}$ is called "collision efficiency". This correcting factor takes into account the fact that, when a droplet is approaching very close to another one, it does not follow a straight line trajectory, but instead is deflected due to its interaction with 


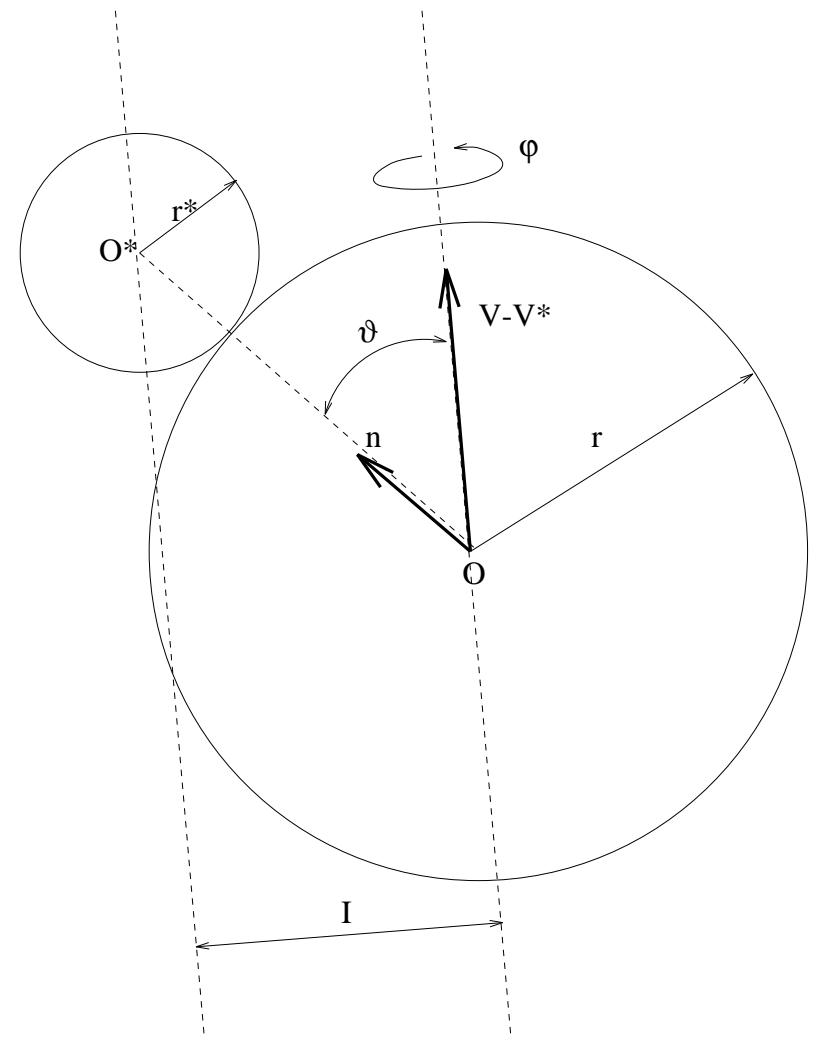

FIG. 3.2. Schematic description of a binary droplet collision

the surrounding gas flow, thus it is possible that the smallest droplet circumvent the largest one and that the collision does not occur.

The determination of the collision efficiency has received the attention of a lot of researchers, because of its importance for the prediction of rain drop formation (see for example $[14,8,2,3]$ or the survey of Villedieu [31]). From a theoretical point of view, the first major contribution is due to Langmuir in [16]. His model relies on the assumption that one of the two colliding droplets is small enough to have a negligible influence on the motion of the other one. Thanks to this hypothesis, Langmuir was able to derive the following expression of the collision efficiency:

$$
E_{\text {coll }}\left(r, r^{*},\left|\mathbf{v}-\mathbf{v}_{*}\right|,|\mathbf{v}-\mathbf{u}|\right)=\frac{1}{1+R / R_{c}} E^{(1)}(K)+\frac{\left(R / R_{c}\right)}{1+R / R_{c}} E^{(2)}(K),
$$

with:

$$
\begin{gathered}
r_{s}=\min \left(r, r_{*}\right), \quad r_{l}=\max \left(r, r_{*}\right), \quad R_{c}=60, \\
R=\frac{\rho_{g}|\mathbf{v}-\mathbf{u}| r_{l}}{\mu_{g}}, \quad K=\frac{2 \rho_{l} r_{s}^{2}\left|\mathbf{v}-\mathbf{v}_{*}\right|}{9 \mu_{g} r_{l}}
\end{gathered}
$$




$$
\begin{aligned}
& \left\{\begin{array}{lr}
E^{(1)}(K)=0 & K \leq 1.214, \\
E^{(1)}(K)=\left[1+\frac{3 \ln (2 K)}{4(K-1.214)}\right]^{-2} & K>1.214,
\end{array}\right. \\
& \begin{cases}E^{(2)}(K)=0 & K \leq 0.0833 \\
E^{(2)}(K)=\frac{K^{2}}{(K+1 / 2)^{2}} & K>0.0833 .\end{cases}
\end{aligned}
$$

The dimensionless parameter $K$ can be seen as the ratio of the stopping distance of the smallest droplet (assuming that its initial relative velocity is $|\mathbf{v}-\mathbf{v} *|$ ) to the radius of the other one. $R$ is the Reynolds number related to the flow arround the largest drop. For small values of $R$, this flow is of viscous type (Stokes analytical solution) and for large values, it is of inviscid type (potential analytical solution). As one would expect, if $K$ is small compared to unity, the collision efficiency goes to zero, whatever the value of the Reynold number, and conversely, if $K$ is large compared to unity, the collision efficiency goes to one.

It is worth noticing that, since the velocity of a small droplet is generally close to the local gas velocity, it is possible to replace the expression of the Reynolds number given above by the following one:

$$
R=\frac{\rho_{g}\left|\mathbf{v}-\mathbf{v}_{*}\right| r_{l}}{\mu_{g}}
$$

The major advantage of this approximation is that the collision efficiency does only depend, then, on the radii and on the relative velocity of the two colliding droplets.

In a more recent study, Beard and Grover [3] have proposed a new expression for the collision efficiency, in order to improve the accuracy of Langmuir's model in the range of intermediate Reynolds numbers. To compute the velocity field around the largest drop, instead of using analytical solutions of simplified models as in Langmuir pioneering work, they used numerical solutions of the full Navier-Stokes equations corresponding to different values of the Reynolds number, covering the range $[0$ 400]. By interpolating the results issued from their calculations, they obtained the following expression for $E_{\text {coll }}$, valid in the range $R \in[0,400]$ :

$$
E_{\text {coll }}(K, R)=\left(\frac{2}{\pi} \operatorname{atan}[\max (H, 0)]\right)^{2}
$$

with:

$$
\left\{\begin{array}{l}
H=0.1465+1.302 Z-0.607 Z^{2}+0.293 Z^{3} \\
Z=\ln \left(K / K_{0}\right) \\
K_{0}=\exp \left(-0.1007-0.358 \ln (R)+0.0261[\ln (R)]^{2}\right)
\end{array}\right.
$$

Figure 3.3 shows a comparison between the two models. As one would expect, they give almost the same results for low and large Reynolds numbers. For intermediate values, according to Beard and Grover calculations, the expression given by Langmuir tends to slightly underestimate the collision efficiency. 


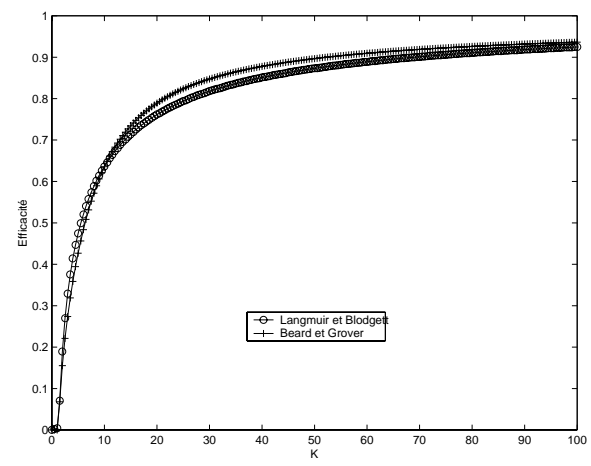

(a) case $R=0.5$

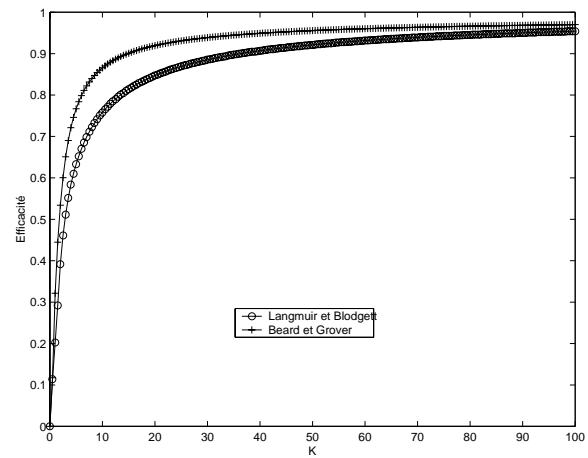

(c) case $R=50$

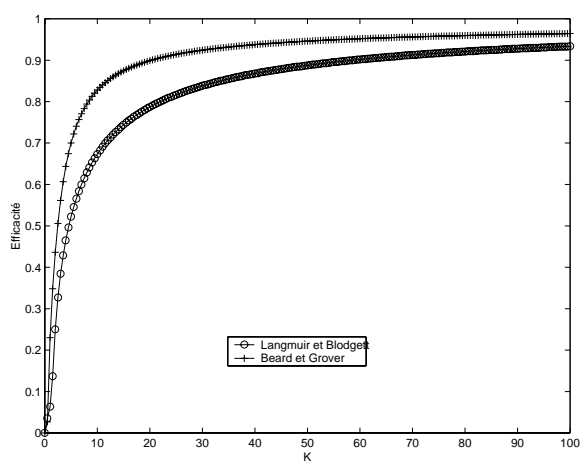

(b) case $R=10$

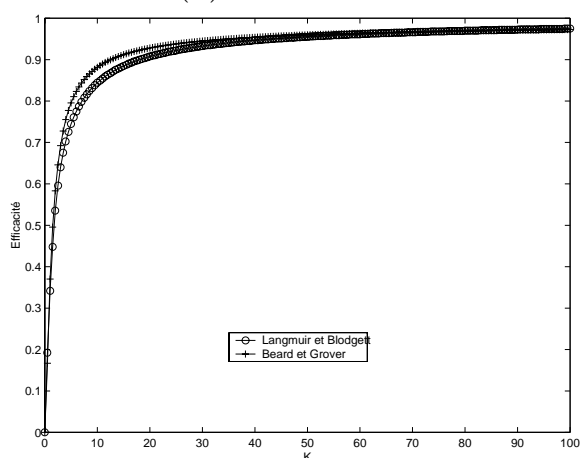

(d) case $R=200$

FIG. 3.3. Comparison between the model proposed by Langmuir with the model proposed by Beard and Grover for the calculation of the collision efficiency

\subsection{Closure assumption}

As mentioned above, to get a closed collision model, it is necessary to find a relation between $f_{p g}^{(2)}$ and $f_{p g}^{(1)}$. Actually, since two colliding droplets are necessarily located at the same space position (droplet radii are assumed to be small compared to all other length scales), it is sufficient to get a relation between $f_{p g}^{(2)}\left(t, \mathbf{x}, \mathbf{v}, \mathbf{u}, r, \mathbf{x}, \mathbf{v}_{*}, \mathbf{u}_{*}\right.$, $\left.r_{*}\right), f_{p g}^{(1)}(t, \mathbf{x}, \mathbf{v}, \mathbf{u}, r)$ and $f_{p g}^{(1)}\left(t, \mathbf{x}, \mathbf{v}_{*}, \mathbf{u}_{*}, r_{*}\right)$.

Let us introduce the joint probability density functions defined as follows:

$$
\begin{gathered}
p_{p g}^{(1)}(t, \mathbf{x}, \mathbf{v}, \mathbf{u}, r)=\frac{1}{n(t)} f_{p g}^{(1)}\left(t, \mathbf{x}, \mathbf{v}_{*}, \mathbf{u}_{*}, r_{*}\right), \\
p_{p g}^{(2)}\left(t, \mathbf{x}, \mathbf{v}, \mathbf{u}, r, \mathbf{x}_{*}, \mathbf{v}_{*}, \mathbf{u}_{*}, r_{*}\right)=\frac{1}{n(t)^{2}} f_{p g}^{(2)}\left(t, \mathbf{x}, \mathbf{v}, \mathbf{u}, r, \mathbf{x}_{*}, \mathbf{v}_{*}, \mathbf{u}_{*}, r_{*}\right),
\end{gathered}
$$

with $n(t)$ being the total number of droplets at time $t$. The definition of the conditionnal probability density function yields:

$$
p_{p g}^{(2)}\left(t, \mathbf{x}, \mathbf{v}_{*}, \mathbf{u}_{*}, r_{*}, \mathbf{x}, \mathbf{v}, \mathbf{u}, r\right)=p_{p g}^{(1 \mid 1)}\left(t, \mathbf{x}, \mathbf{v}_{*}, \mathbf{u}_{*}, r_{*} \mid \mathbf{x}, \mathbf{v}, \mathbf{u}, r\right) p_{p g}^{(1)}(t, \mathbf{x}, \mathbf{v}, \mathbf{u}, r)
$$


with $p_{p g}^{(1 \mid 1)}\left(t, \xi_{*} \mid \xi\right) d \xi_{*}$ being the probability, for a given pair of droplet, to find the first one in the range $\left(\xi_{*}, \xi_{*}+d \xi_{*}\right)$, given that the second one is in $\xi$. Hence, using formal notations, $p_{p g}^{(1 \mid 1)}$ satisfies:

$$
p_{p g}^{(1 \mid 1)}\left(t, \xi_{*} \mid \xi\right) d \xi_{*}=\operatorname{Prob}\left(X_{t}^{1} \in\left(\xi_{*}, \xi_{*}+d \xi_{*}\right) \mid X_{t}^{2}=\xi\right)
$$

where $X_{t}^{1}$ and $X_{t}^{2}$ stand for the first and second droplet position in the phase space at time $t$. At this level, it is necessary to make a physical assumption. With the notations introduced above, the fundamental idea proposed by Simonin and Lavieville in [17] can be written as follows:

$$
\operatorname{Prob}\left(X_{t}^{1} \in\left(\xi_{*}, \xi_{*}+d \xi_{*}\right) \mid X_{t}^{2}=\xi\right) \simeq \operatorname{Prob}\left(X_{t}^{1} \in\left(\xi_{*}, \xi_{*}+d \xi_{*}\right) \mid \mathbf{u}_{g}(t, \mathbf{x})=\mathbf{u}\right) .
$$

From a physical point of view, (3.8) is rather natural. It means that among all the "information" known about the second droplet, the most important is the value of the gas velocity "seen" by this droplet because, for two droplets located in the vicinity of the same point, the knowledge of the gas velocity seen by the second one provides a good estimate of the gas velocity seen by the first one, which in turn is strongly related to its own velocity. We refer to [17] for numerical experiments (based on DNS calculations) to check the validity of this closure hypothesis.

Thus, if $\mathbf{x}_{*}=\mathbf{x}$, the closure assumption (3.8) and the definition of $\mathbf{u}_{t}^{1}$ formally yield:

$\operatorname{Prob}\left(X_{t}^{1} \in\left(\xi_{*}, \xi_{*}+d \xi_{*}\right) \mid X_{t}^{2}=\xi\right)$

$\simeq \operatorname{Prob}\left(\mathbf{x}_{t}^{1} \in\left(\mathbf{x}, \mathbf{x}+d \mathbf{x}_{*}\right), \mathbf{v}_{t}^{1} \in\left(\mathbf{v}_{*}, \mathbf{v}_{*}+d \mathbf{v}_{*}\right), r_{t}^{1} \in\left(r_{*}, r_{*}+d r_{*}\right) \mid \mathbf{u}_{g}(t, \mathbf{x})=\mathbf{u}\right) \delta_{\mathbf{u}_{*}-\mathbf{u}} d \mathbf{u}_{*}$.

Let us introduce the eulerian gas velocity pdf (see Pope [21]) defined as:

$$
\int_{U} p_{g}(t, x, \mathbf{u}) d u=\operatorname{Prob}\left(\mathbf{u}_{g}(t, \mathbf{x}) \in U\right)
$$

In case of an isotropic homogeneous turbulent gas flow, as considered in this paper, one has:

$$
p_{g}(t, \mathbf{x}, \mathbf{u})=\frac{1}{\left(2 \pi \sigma_{g}\right)^{3 / 2}} \exp \left(-\frac{|\mathbf{u}|^{2}}{2 \sigma_{g}^{2}}\right)
$$

If $\xi_{*}=\left(\mathbf{x}, \mathbf{v}_{*}, \mathbf{u}_{*}, r\right)$, using the joint probability instead of the conditional one, (3.9) finally yields:

$$
\begin{aligned}
& \operatorname{Prob}\left(X_{t}^{1} \in\left(\xi_{*}, \xi_{*}+d \xi_{*}\right) \mid X_{t}^{2}=\xi\right) \\
& \simeq \frac{\operatorname{Prob}\left(\mathbf{x}_{t}^{1} \in\left(\mathbf{x}, \mathbf{x}+d \mathbf{x}_{*}\right), \mathbf{v}_{t}^{1} \in\left(\mathbf{v}_{*}, \mathbf{v}_{*}+d \mathbf{v}_{*}\right), r_{t}^{1} \in\left(r_{*}, r_{*}+d r_{*}\right), \mathbf{u}_{t}^{1}(\in)\left(\mathbf{u}, \mathbf{u}+d \mathbf{u}_{*}\right)\right)}{p_{g}(t, \mathbf{x}, \mathbf{u})} \delta_{\mathbf{u}_{*}-\mathbf{u}}
\end{aligned}
$$


Therefore, coming back to the initial notations, it follows from (3.6), (3.7) and (3.11) that:

$$
f_{p g}^{(2)}\left(t, \mathbf{x}, \mathbf{v}_{*}, \mathbf{u}_{*}, r_{*}, \mathbf{x}, \mathbf{v}, \mathbf{u}, r\right) \simeq \frac{f_{p g}^{(1)}\left(t, \mathbf{x}, \mathbf{v}_{*}, \mathbf{u}_{*}, r_{*}\right) f_{p g}^{(1)}(t, \mathbf{x}, \mathbf{v}, \mathbf{u}, r)}{p_{g}(t, \mathbf{x}, \mathbf{u})} \delta_{\mathbf{u}_{*}-\mathbf{u}}
$$

which is the fundamental relation needed to close the collision model.

3.4. Expression of the collision operator. All the modeling assumptions presented in the previous subsections finally lead to the following expression of the collision operator:

$$
Q_{\text {coll }}\left(f_{p g}\right)=-Q^{-}\left(f_{p g}\right)+Q_{\text {coal }}^{+}\left(f_{p g}\right)+Q_{r e f}^{+}\left(f_{p g}\right)
$$

with:

$$
\begin{aligned}
& Q^{-}\left(f_{p g}\right)(t, \mathbf{x}, \mathbf{v}, \mathbf{u}, r)= \\
& \int_{\mathbb{R}^{+}} \int_{\mathbb{R}^{3}} \int_{0}^{\frac{\pi}{2}} \int_{0}^{2 \pi} \frac{B\left(\theta,\left|\mathbf{v}-\mathbf{v}_{*}\right|, r, r_{*}\right)}{p_{g}(t, \mathbf{x}, \mathbf{u})} f_{p g}(t, \mathbf{x}, \mathbf{v}, \mathbf{u}, r) f_{p g}\left(t, \mathbf{x}, \mathbf{v}_{*}, \mathbf{u}, r_{*}\right) d \phi d \theta d \mathbf{v}_{*} d r_{*}, \\
& Q_{r e f}^{+}\left(f_{p g}\right)(t, \mathbf{x}, \mathbf{v}, \mathbf{u}, r)= \\
& \int_{\mathbb{R}^{+}} \int_{\mathbb{R}^{3}} \int_{0}^{\theta_{1}} \int_{0}^{2 \pi} \frac{B\left(\theta,\left|\mathbf{v}^{\prime}-\mathbf{v}_{*}^{\prime}\right|, r, r_{*}\right)}{p_{g}(t, \mathbf{x}, \mathbf{u})(2 e-1)} f_{p g}\left(t, \mathbf{x}, \mathbf{v}^{\prime}, \mathbf{u}, r\right) f_{p g}\left(t, \mathbf{x}, \mathbf{v}_{*}^{\prime}, \mathbf{u}, r_{*}\right) d \phi d \theta d \mathbf{v}_{*} d r_{*}, \\
& Q_{c o a l}^{+}\left(f_{p g}\right)(t, \mathbf{x}, \mathbf{v}, \mathbf{u}, r)=\int_{0}^{r} \int_{\mathbb{R}^{3}} \int_{\theta_{1}}^{\theta_{2}} \int_{0}^{2 \pi} \frac{B\left(\theta,\left|\mathbf{v}_{\diamond}-\mathbf{v}_{*}\right|, r_{\diamond}, r_{*}\right),}{p_{g}(t, \mathbf{x}, \mathbf{u})} \\
& \quad \times \frac{r^{11}}{r_{\diamond}^{11}} f_{p g}\left(t, \mathbf{x}, \mathbf{v}_{\diamond}, \mathbf{u}, r_{\diamond}\right) f_{p g}\left(t, \mathbf{x}, \mathbf{v}_{*}, \mathbf{u}, r_{*}\right) d \phi d \theta d \mathbf{v}_{*} d r_{*},
\end{aligned}
$$

where $\frac{1}{2 e-1}$ is nothing but the jacobian of the mapping $\left(\mathbf{v}, \mathbf{v}_{*}\right) \rightarrow\left(\mathbf{v}^{\prime}, \mathbf{v}_{*}^{\prime}\right), \mathbf{v}^{\prime}$ and $\mathbf{v}_{*}^{\prime}$ being the precollisional velocities in case of a reflexive separation. According to (3.1), they are defined as:

$$
\begin{aligned}
& \mathbf{v}^{\prime}=\mathbf{v}-\frac{2 e}{2 e-1} \frac{r_{*}^{3}}{r^{3}+r_{*}^{3}}\left(\mathbf{v}-\mathbf{v}_{\star}\right) \cdot \mathbf{n} \mathbf{n}, \\
& \mathbf{v}_{\star}^{\prime}=\mathbf{v}_{\star}+\frac{2 e}{2 e-1} \frac{r^{3}}{r^{3}+r_{*}^{3}}\left(\mathbf{v}-\mathbf{v}_{\star}\right) \cdot \mathbf{n} \mathbf{n},
\end{aligned}
$$

with $\mathbf{n}$ being the unit vector directed along the colliding droplet center line. $\theta$ is the angle between $\mathbf{n}$ and the relative velocity $\mathbf{w}^{\prime}$ just before the collision. $\phi$ is the angle between the projection of $\mathbf{n}$ and any fixed direction in a plane orthogonal to $\mathbf{w}^{\prime}$. 
The function $B$ is defined as:

$$
B\left(\theta,|\mathbf{w}|, r, r_{\star}\right)=E_{\text {coll }}\left(|\mathbf{w}|, r, r_{\star}\right)|\mathbf{w}|\left(r+r_{\star}\right)^{2} \cos \theta \sin \theta
$$

$r_{\diamond}$ and $\mathbf{v}_{\diamond}$ denote the precollisional radius and velocity, in the case of a permanent coalescence leading to the formation of a droplet of radius $r$ and velocity $\mathbf{v}$ :

$$
\begin{gathered}
\mathbf{v}_{\diamond}=\frac{r^{3} \mathbf{v}}{r^{3}-r_{*}^{3}}-\frac{r_{*}^{3} \mathbf{v}_{*}}{r^{3}-r_{*}^{3}} \\
r_{\diamond}=\left(r^{3}-r_{*}^{3}\right)^{1 / 3}
\end{gathered}
$$

The expression $\frac{r^{11}}{r_{\diamond}^{11}}$ is the jacobian of the mapping $\left(r \rightarrow r_{\diamond}\right)$, with $r_{*}$ being fixed. Finally, $\theta_{1}$ and $\theta_{2}$ denote the values of $\theta$, which are delimiting the coalescence regime. Using the geometrical definition of the impact parameter, it is easy to check that $\theta_{1}$ and $\theta_{2}$ are related to $b_{1}$ and $b_{2}$, according to the relation $\theta_{i}=\arcsin \left(b_{i}\right)$.

We now conclude this section by few comments on the expression of the collision operator.

- In the expression of $Q\left(f_{p g}\right)$, it is worth noticing that the fluctuating gas velocity $\mathbf{u}$ plays a similar role as the space position $\mathbf{x}$, in the sense that there is no integration with respect to these two variables. This corresponds to the fact that colliding droplets are necessarily located at the same position and are necessarily "seeing" the same turbulent gas velocity. This property may be used to extend classical Monte-Carlo algorithms, used for the Boltzmann equation, to the case of model (3.13) [20].

- If gas and particle velocities are supposed to be uncorrelated, the classical expression of $Q^{-}$, based on the chaos assumption, may be recovered from (3.14). In this case, one has:

$$
f_{p g}(t, \mathbf{x}, \mathbf{v}, \mathbf{u}, r)=f_{p}(t, \mathbf{x}, \mathbf{v}, r) p_{g}(t, \mathbf{x}, \mathbf{u}) .
$$

Substituting this expression in the definition of $Q^{-}\left(f_{p g}\right)$ and integrating with respect to $\mathbf{u}$ yield:

$$
\begin{aligned}
& \bar{Q}^{-}\left(f_{p}\right)(t, \mathbf{x}, \mathbf{v}, r)= \\
& \int_{\mathbb{R}^{+}} \int_{\mathbb{R}^{3}} \int_{0}^{\frac{\pi}{2}} \int_{0}^{2 \pi} B\left(\theta,\left|\mathbf{v}-\mathbf{v}_{*}\right|, r, r_{*}\right) f_{p}(t, \mathbf{x}, \mathbf{v}, r) f_{p}\left(t, \mathbf{x}, \mathbf{v}_{*}, r_{*}\right) d \phi d \theta d \mathbf{v}_{*} d r_{*}
\end{aligned}
$$

which is the usual expression of $Q^{-}[25,30]$.

- The generalized chaos assumption would correspond to the following closure relation:

$$
f_{p g}^{(2)}\left(t, \mathbf{x}, \mathbf{v}, \mathbf{u}, r, \mathbf{x}_{*}, \mathbf{v}_{*}, \mathbf{u}_{*}, r_{*}\right)=f_{p g}^{(1)}(t, \mathbf{x}, \mathbf{v}, \mathbf{u}, r) f_{p g}^{(1)}\left(t, \mathbf{x}_{*}, \mathbf{v}_{*}, \mathbf{u}_{*}, r_{*}\right),
$$

and the corresponding expression of $Q^{-}$would be then:

$$
\begin{aligned}
Q_{\text {chaos }}^{-}\left(f_{p g}\right)( & t, \mathbf{x}, \mathbf{v}, \mathbf{u}, r)=\int_{\mathbb{R}^{+}} \int_{\mathbb{R}^{3}} \int_{0}^{\frac{\pi}{2}} \int_{0}^{2 \pi} B\left(\theta,\left|\mathbf{v}-\mathbf{v}_{*}\right|, r, r_{*}\right) \\
& \times f_{p g}(t, \mathbf{x}, \mathbf{v}, \mathbf{u}, r) f_{p g}\left(t, \mathbf{x}, \mathbf{v}_{*}, \mathbf{u}_{*}, r_{*}\right) d \phi d \theta d \mathbf{v}_{*} d \mathbf{u}_{*} d r_{*} .
\end{aligned}
$$


By integrating this expression with respect to $\mathbf{u}$ and $\mathbf{u}_{*}$, we recover again the usual expression of $\bar{Q}^{-}\left(f_{p}\right)$ given above.

\section{Asymptotic model for dilute sprays}

In this section, we consider the case of dilute sprays, in which the mean collision time is large with respect to the typical time scale of the turbulence. We show that it is then possible to derive a simplified asymptotic model of Smoluchovski type [28], that can be used to provide some reference numerical solutions.

4.1. Dimensionless joint density equation. Let us introduce the following rescaled variables and dimensionless numbers:

$$
\begin{gathered}
\bar{u}=\frac{u}{\sigma_{g}}, \quad \overline{u_{g}},=\frac{u_{g}}{\sigma_{g}}, \quad \bar{v}=\frac{v}{\sigma_{g}}, \quad \bar{r}=\frac{r}{r_{0}}, \\
\bar{t}=\frac{t}{\tau_{c}}, \quad S(r)=\frac{\tau_{p}}{\tau_{g}}=\frac{2 \rho_{p} r^{2}}{9 \mu_{g} \tau_{g}}, \quad K=\frac{\tau_{c}}{\tau_{g}},
\end{gathered}
$$

with $r_{0}$ being the typical scale of droplet radii, $\tau_{c}$ the collision time defined as $\tau_{c}=$ $\frac{1}{n_{0} r_{0}^{2} \sigma_{g}}, \mathrm{~K}$ the Knudsen number, and $S(r)$ the Stokes number.

For the sake of simplicity, we restrict our attention to the case of spatially homogeneous sprays. Hence the $\mathbf{x}$ variable will be omitted everywhere. Using the notations defined above, the rescaled joint pdf equation writes:

$$
\partial_{t} \overline{f_{p g}}+K D\left(\overline{f_{p g}}\right)=Q\left(\overline{f_{p g}}\right)
$$

where $\overline{f_{p g}}$ stands for the dimensionless joint pdf

$$
\overline{f_{p g}}(t, \bar{v}, \bar{u}, \bar{r})=\frac{1}{n_{0} r_{0} \sigma_{g}^{6}} \overline{f_{p g}}\left(t, \sigma_{g} \bar{v}, \sigma_{g} \bar{u}, r_{0} \bar{r}\right),
$$

and $D$ is the second order differential operator defined as

$$
D(f)=\operatorname{div}_{\bar{v}}\left(\frac{\overline{\mathbf{u}}-\overline{\mathbf{v}}}{S(r)} f\right)-\operatorname{div}_{\bar{u}}\left(\bar{u} f+\nabla_{\bar{u}} f\right) .
$$

4.2. Expression of the asymptotic model. In this section, for the sake of clarity, we drop all the bars. Let us suppose that $K \rightarrow+\infty$, then formally, up to some terms of order $1 / K$, the joint distribution function must satisfy the equation:

$$
D\left(f_{p g}\right)=0,
$$

which means that the droplet "agitation" motion is in equilibrium with the turbulent gas motion. The only positive solution to equation (4.2), under the constraint

$$
\int_{\mathbb{R}^{6}} f_{p g}(t, r, \mathbf{v}, \mathbf{u}) d \mathbf{v} d \mathbf{u}=n(t, r)
$$


writes:

$$
f_{p g}(t, r, \mathbf{v}, \mathbf{u})=n(t, r) M(\mathbf{v}, \mathbf{u}, r)
$$

with:

$$
M(\mathbf{v}, \mathbf{u}, r)=\left[\frac{(1+S(r))}{2 \pi \sqrt{S(r)}}\right]^{3} \exp \left[\frac{(1+S(r))}{2 S(r)}\left(\mathbf{u}^{2}+(1+S(r)) \mathbf{v}^{2}-2 u . v\right)\right] .
$$

The proof of this result can be found in [7]. For completeness, we only indicate here that uniqueness results from the following entropy estimate:

$$
\begin{gathered}
\int_{\mathbb{R}^{6}} D(f)(1+\ln (f)) d \mathbf{v} d \mathbf{u}=\int_{\mathbb{R}^{6}} 4 M\left(\nabla_{u} \sqrt{\frac{f}{M}} \cdot \nabla_{u} \sqrt{\frac{f}{M}}\right) d \mathbf{v} d \mathbf{u} \\
+\frac{1+S}{S}\left[3 \int_{\mathbb{R}^{6}} f d \mathbf{v} d \mathbf{u}-\int_{\mathbb{R}^{6}} \frac{1+S}{S}|\mathbf{v}-\mathbf{u}|^{2} f d \mathbf{v} d \mathbf{u}\right]
\end{gathered}
$$

Integrating equation (4.1) with respect to $\mathbf{u}$ and $\mathbf{v}$, it follows:

$$
\partial_{t} n=\int_{\mathbb{R}^{6}} Q\left(f_{p g}\right) d \mathbf{v} d \mathbf{u}
$$

Hence, replacing $f_{p g}$ by its expression given by (4.3), we obtain that the droplet density $n(t, r)$ is solution of the following coagulation equation of Smoluchowsky type:

$$
\partial_{t} n=\tilde{Q}(n) \quad \text { with } \quad \tilde{Q}(n)=\int_{\mathbb{R}^{6}} Q(n M) d \mathbf{v} d \mathbf{u} .
$$

We can now stand our main result.

Proposition 4.1.

When $K \rightarrow+\infty$, if the collision operator is defined by (3.13)- (3.14)-(3.15)(3.16), then the solution of the dimensionless kinetic equation (4.1) converges formally to the following equilibrium solution:

$$
f_{p g}(t, r, \mathbf{v}, \mathbf{u})=n(t, r) M(\mathbf{v}, \mathbf{u}, r)
$$

where $M$ is defined as

$$
M(\mathbf{v}, \mathbf{u}, r)=\left[\frac{(1+S(r))}{2 \pi \sqrt{S(r)}}\right]^{3} \exp \left[\frac{(1+S(r))}{2 S(r)}\left(\mathbf{u}^{2}+(1+S(r)) \mathbf{v}^{2}-2 \mathbf{u . v}\right)\right]
$$

and $n$ is solution to the coagulation equation

$$
\frac{\partial n}{\partial t}=-\int_{0}^{+\infty} J\left(r, r_{*}\right) n(t, r) n\left(t, r_{*}\right) d r_{*}+\int_{0}^{r} J\left(r_{\diamond}, r_{*}\right) \frac{r^{2}}{r_{\diamond}^{2}} n\left(t, r_{\diamond}\right) n\left(t, r_{*}\right) d r_{*}
$$


with:

$$
J\left(r, r_{*}\right)=\sqrt{2 \pi}\left(r+r_{*}\right)^{2} G\left(r, r_{*}\right) \int_{0}^{+\infty} \xi^{3} E_{\text {coal }}\left(r, r_{*}, G\left(r, r_{*}\right) \sigma_{g} \xi\right) \exp \left(\frac{-\xi^{2}}{2}\right) d \xi,
$$

where $G\left(r, r_{*}\right)$ is defined as:

$$
G\left(r, r_{*}\right)=\sqrt{\frac{S(r)+S\left(r_{*}\right)}{(1+S(r))\left(1+S\left(r_{*}\right)\right)}}
$$

and $E_{\text {coal }}$ is the coalescence efficiency defined as:

$$
E_{\text {coal }}\left(r, r_{*}, w\right)=E_{\text {coll }}\left(r, r_{*}, w\right)\left[b_{2}^{2}(W e, \Delta)-b_{1}^{2}(W e, \Delta)\right]
$$

Remarks:

- If, instead of (3.12), the expression of the collision operator is based on the closure relation (3.19) (generalized chaos assumption), the result of proposition 4.1 still holds but the expression of $G\left(r, r_{*}\right)$ must be replaced by:

$$
G\left(r, r_{*}\right)=\sqrt{\frac{2+S(r)+S\left(r_{*}\right)}{(1+S(r))\left(1+S\left(r_{*}\right)\right)}}
$$

- If $E_{\text {coal }}$ is assumed to be a given constant, it is possible to compare explicitely the coagulation rates corresponding to the closure assumptions (3.12) and (3.19). For small droplets, the first one yields:

$$
J\left(r, r_{*}\right) \simeq C\left(r+r_{*}\right)^{2} \sqrt{r^{2}+r_{*}^{2}},
$$

while hypothesis (3.19) gives:

$$
J\left(r, r_{*}\right) \simeq C^{\prime}\left(r+r_{*}\right)^{2},
$$

with $C$ and $C^{\prime}$ being two constants. As one should expect, the model based on the chaos assumption leads to the largest collision rate. It is worth noticing that the expression of the coagulation rate proposed by Williams and Crane in [32] behaves exactly as (4.5) for small droplets $\left(S \ll 1\right.$ and $\left.S_{*} \ll 1\right)$.

Proof of proposition 4.1. Formally, it has been shown above that $n(t, r)$ solves the following equation:

$$
\frac{\partial n}{\partial t}(t, r)=\int_{\mathbb{R}^{6}} Q\left(f_{p g}\right)(t, r, \mathbf{v}, \mathbf{u}) d \mathbf{v} d \mathbf{u},
$$

with $f_{p g}^{\star}(t, r, \mathbf{v}, \mathbf{u})=n(t, r) M(\mathbf{v}, \mathbf{u}, r), M$ being defined as (4.4). Using that the droplet number is preserved by stretching and reflexive separations, equation (4.7) yields: 


$$
\begin{aligned}
\partial_{t} n(t, r)= & -\int_{\mathbb{R}^{6}} \int_{\mathbb{R}^{+}} \int_{\mathbb{R}^{3}} \int_{\theta_{1}}^{\theta_{2}} \int_{0}^{2 \pi} \frac{B\left(\theta, \sigma_{g}\left|\mathbf{v}-\mathbf{v}_{*}\right|, r, r_{*}\right)}{p_{g}(\mathbf{u})} \\
& \times f_{p g}(t, \mathbf{v}, \mathbf{u}, r) f_{p g}\left(t, \mathbf{v}_{*}, \mathbf{u}, r_{*}\right) d \phi d \theta d \mathbf{v}_{*} d r_{*} d \mathbf{u} d \mathbf{v} \\
& +\int_{\mathbb{R}^{6}} \int_{0}^{r} \int_{\mathbb{R}^{3}} \int_{\theta_{1}}^{\theta_{2}} \int_{0}^{2 \pi} \frac{B\left(\theta, \sigma_{g}\left|\mathbf{v}_{\diamond}-\mathbf{v}_{*}\right|, r_{\diamond}, r_{*}\right)}{p_{g}(\mathbf{u})} \frac{r^{11}}{r_{\diamond}^{11}} f_{p g}\left(t, \mathbf{v}_{\diamond}, \mathbf{u}, r_{\diamond}\right) \\
& \times f_{p g}\left(t, \mathbf{v}_{*}, \mathbf{u}, r_{*}\right) d \phi d \theta d \mathbf{v}_{*} d r_{*} d \mathbf{u} d \mathbf{v},
\end{aligned}
$$

where:

$$
p_{g}(\mathbf{u})=\frac{1}{(2 \pi)^{3 / 2}} \exp \left(-\frac{|\mathbf{u}|^{2}}{2}\right) .
$$

After a short calculation, it follows:

$$
\begin{aligned}
\frac{\partial n}{\partial t}(t, r) & =-\int_{0}^{+\infty} \pi\left(r+r_{*}\right)^{2} Z\left(r, r_{*}\right) n(t, r) n\left(t, r_{*}\right) d r_{*} \\
& +\int_{0}^{r} \pi\left(r_{\diamond}+r_{*}\right)^{2} Z\left(r_{\diamond}, r_{*}\right) \frac{r^{2}}{r_{\diamond}^{2}} n\left(t, r_{\diamond}\right) n\left(t, r_{*}\right) d r_{*}
\end{aligned}
$$

where $Z$ is defined as:

$$
Z\left(r, r_{*}\right)=\int_{\mathbb{R}^{9}}\left|\mathbf{v}-\mathbf{v}_{*}\right| E_{\text {coal }}\left(r, r_{*}, \sigma_{g}\left|\mathbf{v}-\mathbf{v}_{*}\right|\right) \frac{M(\mathbf{v}, \mathbf{u}, r) M\left(\mathbf{v}_{*}, \mathbf{u}, r_{*}\right)}{p_{g}(\mathbf{u})} d \mathbf{v}_{*} d \mathbf{v} d \mathbf{u} .
$$

After integration with respect to $\mathbf{u}$, it follows:

$Z\left(r, r_{*}\right)=C \int_{\mathbb{R}^{6}}\left|\mathbf{v}-\mathbf{v}_{*}\right| E_{\text {coal }}\left(r, r_{*}, \sigma_{g}\left|\mathbf{v}-\mathbf{v}_{*}\right|\right) \exp \left(\frac{-\mathbf{v}^{2}}{2 \alpha^{2}}-\frac{\mathbf{v}_{*}^{2}}{2 \alpha_{*}^{2}}+\chi \chi \frac{\mathbf{v} \cdot \mathbf{v}_{*}}{\alpha \alpha_{*}}\right) d \mathbf{v}_{*} d \mathbf{v}$

with the following notations:

$$
\begin{gathered}
C=\frac{\left(1-\chi^{2} \chi_{*}^{2}\right)^{3 / 2}}{8 \pi^{3} \alpha^{3} \alpha_{*}^{3}}, \\
\chi=\frac{1}{\sqrt{1+S(r)},} \quad \chi_{*}=\frac{1}{\sqrt{1+S\left(r_{*}\right)}}, \\
\alpha=\left(1-\chi^{2} \chi_{*}^{2}\right)^{1 / 2} \chi, \quad \alpha_{*}=\left(1-\chi^{2} \chi_{*}^{2}\right)^{1 / 2} \chi_{*} .
\end{gathered}
$$

Finally, integrating with respect to $\mathbf{v}$ and $\mathbf{v}_{*}$, one obtains: 


$$
Z\left(r, r_{*}\right)=\sqrt{\frac{2}{\pi}} G\left(r, r_{*}\right) \int_{0}^{+\infty} \xi^{3} E_{\text {coal }}\left(r, r_{*}, G\left(r, r_{*}\right) \sigma_{g} \xi\right) \exp \left(\frac{-\xi^{2}}{2}\right) d \xi
$$

with:

$$
G\left(r, r_{*}\right)=\sqrt{\frac{S(r)+S\left(r_{*}\right)}{(1+S(r))\left(1+S\left(r_{*}\right)\right)}}
$$

which is the expected result. Q.E.D.

\section{Conclusion}

In this paper, we have presented an original model for droplet coalescence in homogeneous turbulent sprays, which takes into account the correlations induced by the effect of the gas on the droplet motion. The key point of our approach consists of using the joint distribution function, $f_{p g}^{(1)}(t, \mathbf{x}, \mathbf{v}, \mathbf{u}, r)$, instead of the simple one, $f_{p}^{(1)}(t, \mathbf{x}, \mathbf{v}, r)$. In the case of a dilute spray, for which the mean collision time is large with respect to the typical time scale of the turbulence, we have derived an asymptotic model, of Smoluchovski type, that could be used to provide reference numerical solutions, which in turn could be compared with results issued from direct numerical simulations. This work is currently in progress.

\section{REFERENCES}

[1] N. Ashgriz and J.Y. Poo, Coalescence and separation in binary collisions of liquid drops, Journal of Fluid Mechanics, 221:183-204, 1990.

[2] K.V. Beard and S.N. Grover, Numerical collision efficiencies for small raindrops colliding with micron size particles, Journ. of Atmospheric Sciences, 31:543-550, 1974.

[3] K.V. Beard and H.R. Pruppacher, An experimental test of the theoritically calculated collision efficiency of cloud drops, Journ. of Geophys. Res., 73:6407-6414, 1968.

[4] P.R. Brazier-Smith, S.G. Jennings, and J. Latham, The interaction of falling water drops: coalescence, Proceedings R. Soc. London, 326:393-408, 1972.

[5] G.T. Csanady, Turbulent diffusion of heavy particles in the atmosphere, J. Atmos. Sci., 20:201208, 1963.

[6] J.F. Clouet and K. Domelevo, Solution of a kinetic stochastic equation modeling a spray in a turbulent gas flow, Math. Models Methods Appl. Sci., 7:239-263, 1997.

[7] K. Domelevo and P. Villedieu, Work in preparation, 2003.

[8] R.H. Davis, Rate of coagulation of a dilute polydisperse system of sedimenting spheres, Journal of Fluid Mechanics, 145:379-408, 1984.

[9] E. Deutsch and O. Simonin, Large eddy simulation applied to the motion of particles in stationary homogeneous fluid turbulence, FED-vol., 110:255-260, ASME, 1991.

[10] J.P. Estrade, H. Carentz, G. Lavergne, and Y. Biscos, Experimental investigation of binary collision of ethanol droplets - a model for droplet coalescence and bouncing, Int. Journ. of Mult. Ph. Flows, 20:486-491, 1999.

[11] P. Fevrier, Etude numérique des effets de concentration préférentielle et de corrélation spatiale entre vitesses de particules solides en turbulence homogène isotrope stationnaire, PHD Thesis, INP Toulouse, 2000.

[12] M. Gavaises, A. Theodorakakos, G. Bergeles, and G. Breen, Evaluation of the effect of droplet collisions on spray mixing, Proc. of the Inst. of Mech. Eng., 210:465-475, 1996.

[13] T. Goudon and F. Poupaud, preprint, 2003.

[14] L.M. Hocking and P.R. Jonas, The collision efficiency of small drops, Quart J. R. Met. Soc., 96:722-729, 1970.

[15] J. Hylkema, Modélisation cinétique et simulation numérique d'un brouillard dense de gouttelettes - Application aux propulseur à poudre, PHD Thesis, SUPAERO, 1998. 
[16] I. Langmuir, The production of rain by a chain reaction in cumulous clouds at temperatures above freezing, J. Meteor., 5:175-192, 1948.

[17] J. Laviéville, E. Deutsch, and O. Simonin, Large eddy simulation of interactions between colliding particles and a homogeneous isotropic turbulence field, ASME FED-288:347-357, 1995.

[18] J.P. Minier and J. Pozorski, Analysis of existing lagrangian models and new propositions for particle dispersion in homogeneous stationary turbulence, Technical Report EDF, HE44/92.29, 1992.

[19] J.P. Minier and E. Peirano, The pdf approach to turbulent polydispersed two-phase flows, Physics Report, 352:1-214, 2001.

[20] M. Moreau, P. Fede, O. Simonin, and P. Villedieu, Monte Carlo Simlulation of colliding particles suspended in gas-solid homogeneous turbulent shear flows, Proc. of FEDSM03, 4th ASME-JSME Joint Fluids Engineering Conference, Honolulu, USA, July 6-10, 2003.

[21] S.B. Pope, Lagrangian pdf methods for turbulent flows, Annual Review of Fluid Mechanics, 26:23-63, 1994.

[22] S.L. Post and J. Abraham, Modeling the outcome of drop-drop collisions in Diesel sprays, Int. Journ. of Mult. Ph. Flows, 28:997-1019, 2002.

[23] J. Pozorsky, J.P. Minier, and O. Simoni, Analysis and new propositions for the crossingtrajectory effect in lagrangian turbulent dispersion model, FED-vol:166, Gas Solid Flows, ASME, 1993.

[24] Reeks On a kinetic equation for the transport of particles in turbulent flows, Phys. Fluids A, 3(3), 1991.

[25] P.J. O'Rourke, Collective drop effects on vaporizing liquid sprays, PHD thesis, Los Alamos National Laboratory, 1981.

[26] P.J. O'Rourke and F. Bracco, Modeling of drop interactions in thick sprays and a comparison with experiments, Proc. of the Inst. of Mech. Eng., 9:101-106, 1980.

[27] O. Simonin, Continuum modelling of dispersed two-phase flows, Combustion and turbulence in two-phase flows, Lecture Series 1996-02 - von Karman Institute for fluid dynamics, 1996.

[28] M.V. Smoluchovski, Versuch einer mathematischen Theorie der koagulationskinetik kolloider Lsungen, Z. Phys. Chem., 92:129-168, 1917.

[29] Rger, Hohmann, Sommerfeld, and Kohnen, Euler - Lagrange calculations of turbulent sprays : the effect of droplet collisions and coalescence, Atomization and Sprays, 10-1, 2000.

[30] P. Villedieu and J. Hylkema A random particle method based on a kinetic equation for the numerical simulation of dense sprays of liquid droplets, Notes aux CRAS, Srie I Mathmatiques, t. 325-3:323-328, 1997.

[31] P. Villedieu Etude bibliographique sur la modélisation des collisions de gouttelettes, ONERA Tech. Report, DTIM 1.3783.00, 1998.

[32] J.J.E. Williams and R.I. Crane, Particle coagulation rate in turbulent flows, Int. J. Multiphase Flows, 9(4):421-435, 1983.

[33] J.D. Woods and B.J. Mason, Experimental determination of collection efficiencies for small water droplets in air, Quart J. R. Met. Soc., 90:373-381, 1964. 